\title{
METODOLOGIA DE CARACTERIZAÇÃO DO MODELO DE VISCOSIDADE DE MATERIAIS PARA SIMULAÇÃO VIRTUAL DE COMPONENTES PLÁSTICOS
}

\author{
Renan Melhado Mazza ${ }^{1}$, Cleber Pagliosa ${ }^{1}$ e Fabio Caires ${ }^{1}$ \\ ${ }^{1}$ Grupo Smarttech
}

E-mails: renan.melhado@ smarttech.com.br, cleber.pagliosa@smarttech.com.br, fabio.caires@smarttech.com.br

\section{RESUMO}

A pressão exercida pelas montadoras sobre os fornecedores para reduzir prazos e custos dos desenvolvimentos criou a necessidade de aumentar o uso de programas CAE como ferramenta auxiliar no desenvolvimento. Um dos principais gargalos nesses estudos são a confiabilidade e a disponibilidade das informações da matéria-prima. Muitas vezes essas informações não são encontradas e quando estão disponíveis são antigas e/ ou não representam a condição atual, por exemplo, adição de material reciclado. Esse estudo avalia uma possível alternativa para caracterização dos coeficientes da equação WLF para análise de injeção de materiais poliméricos. A metodologia consiste em obter os coeficientes do modelo de viscosidade, utilizando a curva de pressão obtida na máquina injetora para diferentes cenários e, à partir de um estudo estatístico dos resultados obtidos em diversas simulações de injeção. Os principais ganhos com o uso dessa metodologia em comparação com a caracterização em laboratório são a utilização de dados do processo fabril, considerando fatores antes não avaliados como, mão de obra, ambiente e máquina injetora. Desta forma, a metodologia proposta possibilita obter dados robustos minimizando a dependência dos dados de materiais.

\section{INTRODUÇÃO}

Com o aumento da competitividade global e incentivos do governo para empresas que buscarem o aumento na eficiência energética de veículos automotores, como o programa Inova-Auto [1], aumenta a busca por redução de peso e custo no desenvolvimento de novos projetos. Diminuição do peso em novos projetos resulta em maior utilização de material plástico no desenvolvimento de novos veículos, principalmente na conversão de peças metálicas para plásticas, ou na otimização do peso das peças que já são fabricadas com esse tipo de material.

Entre os processos de fabricação de peças plásticas o mais utilizado é o processo de moldagem por injeção. A predominância na utilização desse processo se dá pela alta repetibilidade e produtividade, em virtude dos tempos necessários para moldagem serem relativamente baixos [2]. Esse cenário tem ampliado a utilização de simulação numérica de injeção, além de haver um aumento na expectativa de qualidade e correlação dos resultados com o processo real.

Entre as principais variáveis que afetam o processo e consequentemente a qualidade das informações obtidas na simulação estão as propriedades materiais [3,4], cuja complexidade de caracterização dificulta a obtenção das informações, além disso, nem sempre o material a ser utilizado está disponível no banco de dados do programa comercial utilizado [5]. Outro ponto 
é que quando essa informação está acessível nem sempre é atualizada, o que causa um erro no resultado obtido da simulação.

O objetivo desse trabalho é avaliar a viabilidade de uma metodologia para se obter as propriedades materiais para simulação do processo de injeção de plástico, através das seguintes fontes:

- Materiais similares que estejam disponíveis no banco de dados;

- Automação do processo de simulação;

- Dados possíveis de serem obtidos no chão de fábrica;

- Métodos de otimização e delineamento de experimentos.

Neste estudo, a análise de injeção foi realizada utilizando o programa Autodesk Simulation Moldflow Insight 2014 e, a automação e estudo dos experimentos foi realizada utilizado o programa Isight.

\section{METODOLOGIA}

A partir de um caso real foi realizado o processo inverso para se obter as características do material para o processo de injeção. A figura 1 apresenta as peças selecionadas para o estudo:
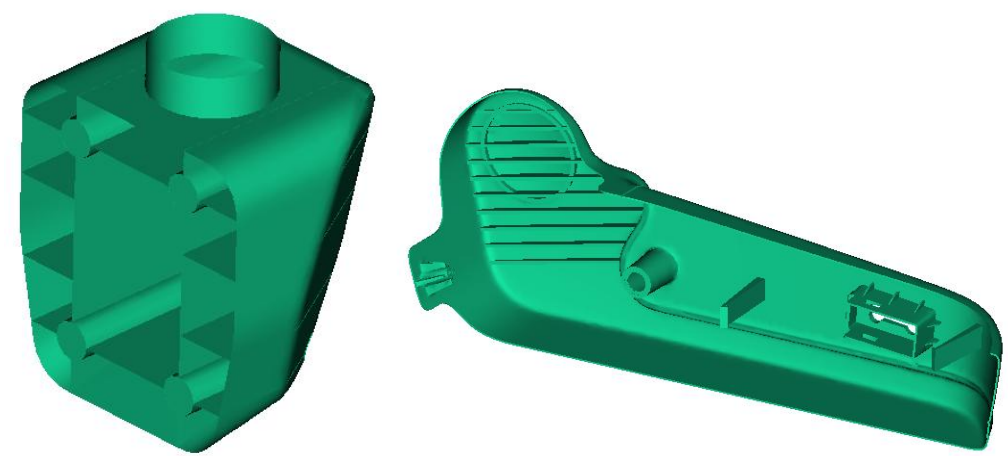

Figure 1. Peças utilizadas no estudo, "retrovisor" e "lateral de banco"

Ambas as peças utilizam a mesma matéria-prima, Moplen HP400M da empresa Basell Polyolefins Europe, esta já está caracterizada e disponível no banco de dados do programa. A viscosidade do material é descrita pelo gráfico de viscosidade em função da taxa de cisalhamento para diferentes temperaturas conforme a figura 2.

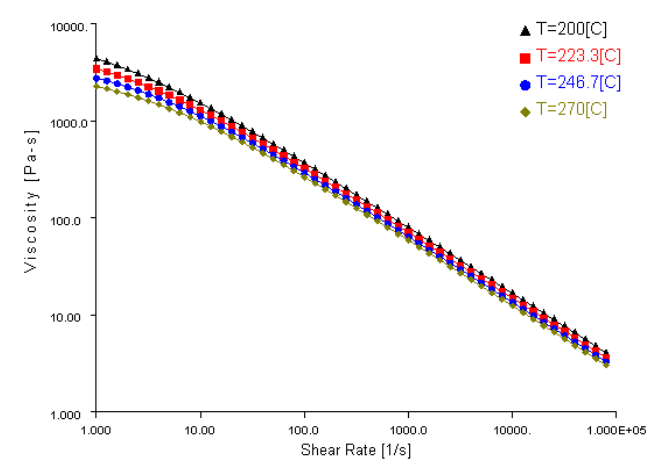

Figure 2. Gráfico de viscosidade do material Moplen HP400M 
Esse gráfico de viscosidade pode ser obtido via ensaio em laboratório utilizando reômetro acoplado a uma de máquina de injeção, conforme norma ISO 11443:2005 ou ASTM D3853508. A partir desse ensaio são obtidos alguns coeficientes que serão utilizados pelo algoritmo do programa para calcular o escoamento do material, ver figura 3.

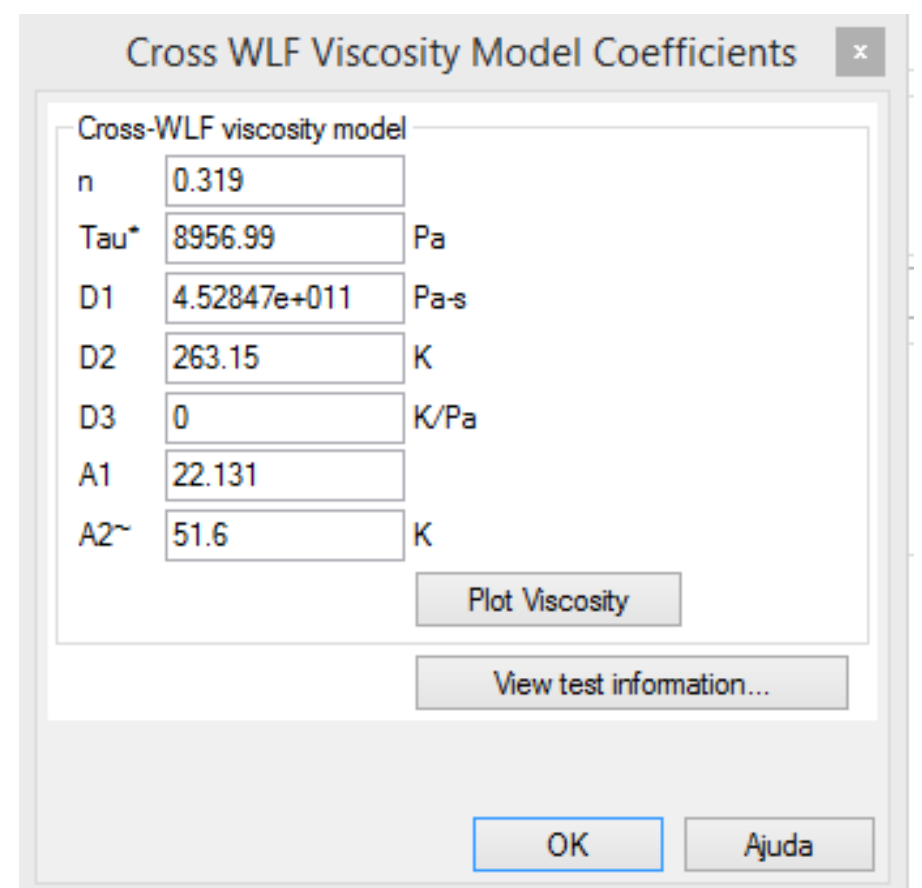

Figure 3. Coeficientes do modelo de viscosidade CROSS-WLF do material Moplen HP400M

Uma vez que em um caso real essa informação será desconhecida, utilizou-se neste estudo as propriedades estimadas do material. Então um processo numérico de calibração destas propriedades deverá resultar em valores que posteriormente serão comparados aos coeficientes obtidos experimentalmente.

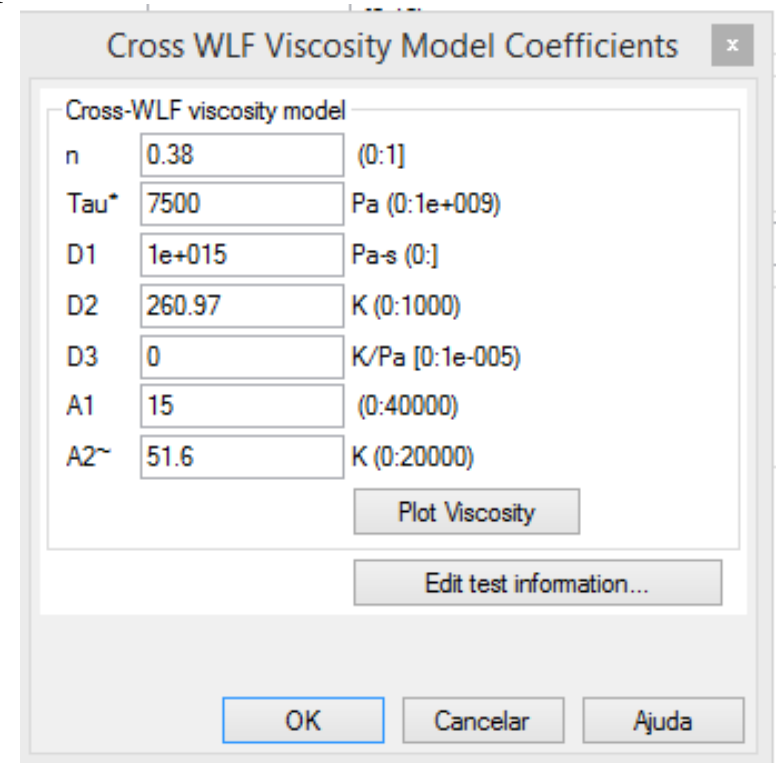

Figure 4. Coeficientes do modelo de viscosidade CROSS-WLF do material inicial do estudo.

Para definir os objetivos da calibração, utilizou-se os gráficos de pressão de injeção que podem ser obtidos em alguns modelos de máquina injetora. 


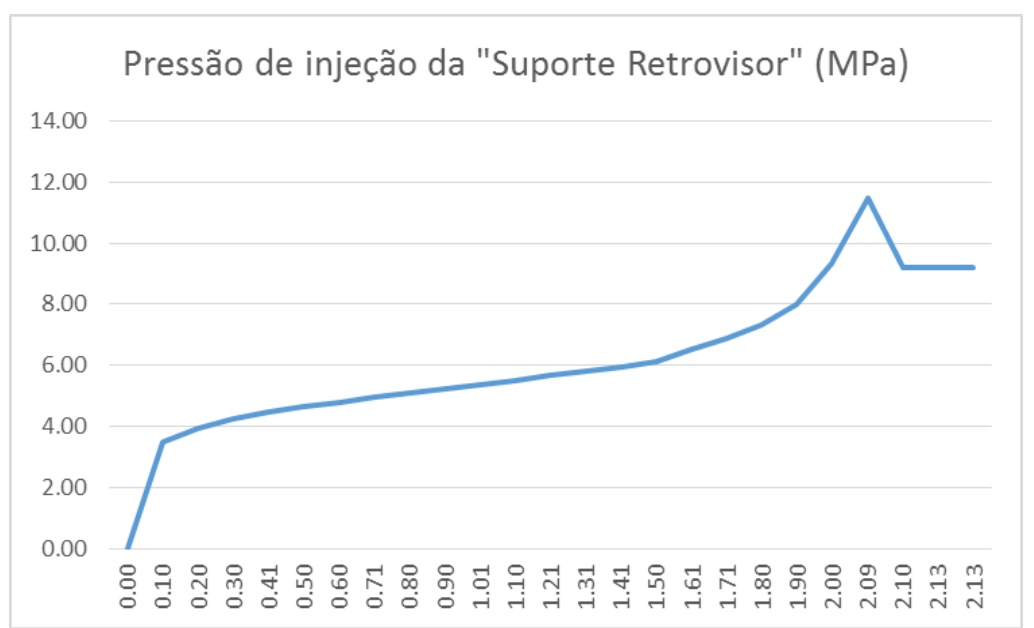

Figure 5. Gráfico de pressão do processo de injeção da peça "retrovisor"

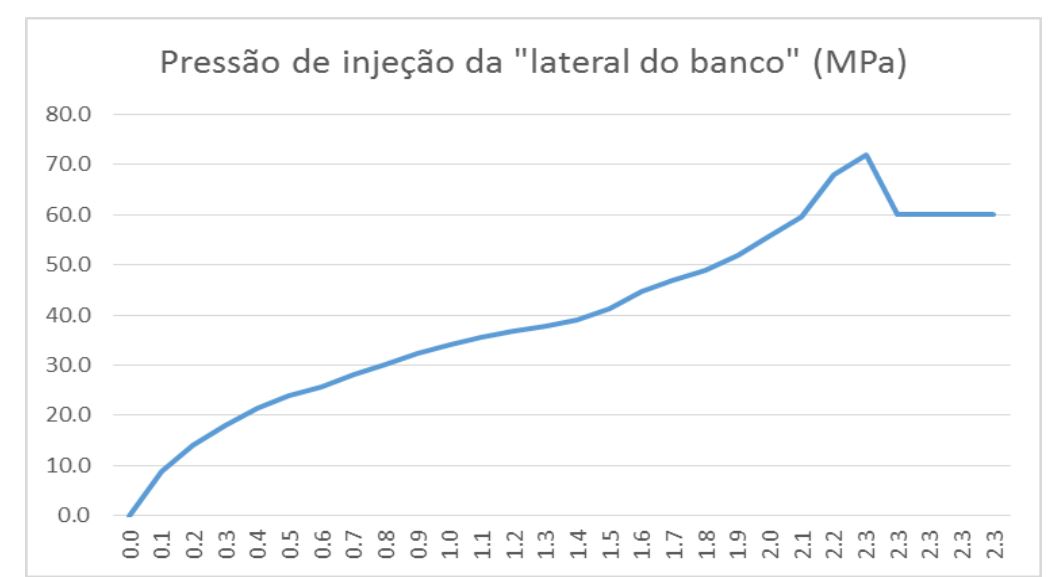

Figure 6. Gráfico de pressão do processo de injeção da peça "lateral de banco"

\subsection{Criação do fluxo automatizado de simulação}

Para realização da calibração foram executadas diversas simulações de injeção utilizando o programa comercial Autodesk Simulation Moldflow Insight 2014 (ASMI). Para a realização dessas análises de maneira automatizada, foi gerado um executável que altera o modelo do ASMI (*.sdy), submete ao cálculo da solução e extrai os resultados, entre eles o gráfico de pressão que será comparado com o gráfico de referência.

Para gerenciar este processo de simulação foi utilizado o programa comercial Simulia Isight 5.8. Com ele foi possível criar um fluxo com todas as etapas de análise e comparação das curvas de resposta. 


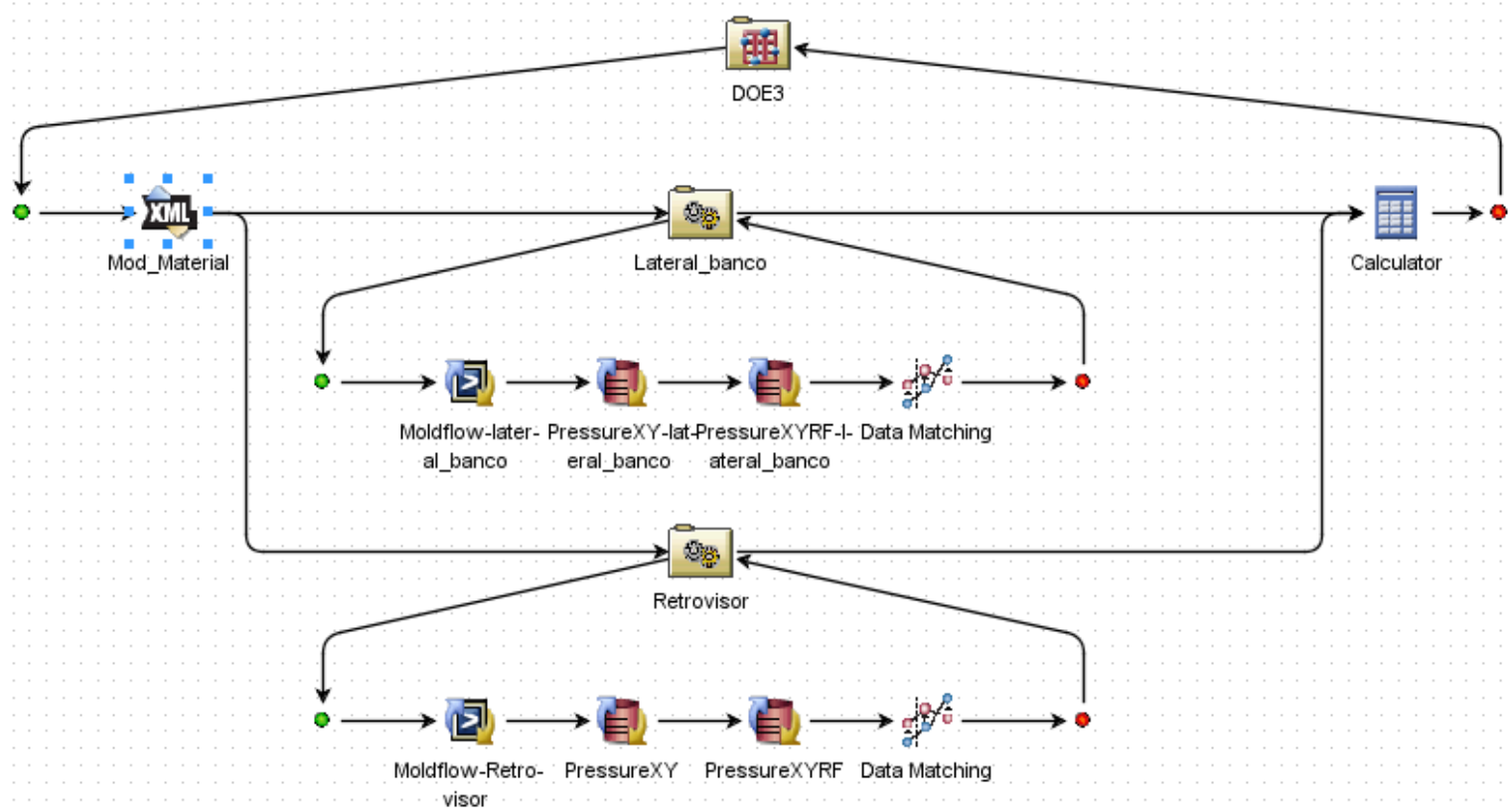

Figure 7. Tela do programa Isight - workflow do processo de calibração de modelo.

O fluxo é separado em componentes, o primeiro componente é o "mold_material", nesse componente são alteradas as propriedades do material que irá alimentar os fluxos de análise das duas peças. Os fluxos de análise são iguais e compostos por um primeiro componente "Moldflow" que executa o programa ASMI e exporta o gráfico de pressão. Após, são utilizados dois componentes de leitura de arquivo de texto: o primeiro lê o gráfico de pressão a partir do resultado da análise e o segundo componente carrega o gráfico de pressão utilizado como referência. Por fim os gráficos são comparados utilizando dois critérios no componente "Data Matching". Os dois critérios utilizados foram fator de correlação entre os dois gráficos e a soma do quadrado da diferença das áreas dos gráficos.

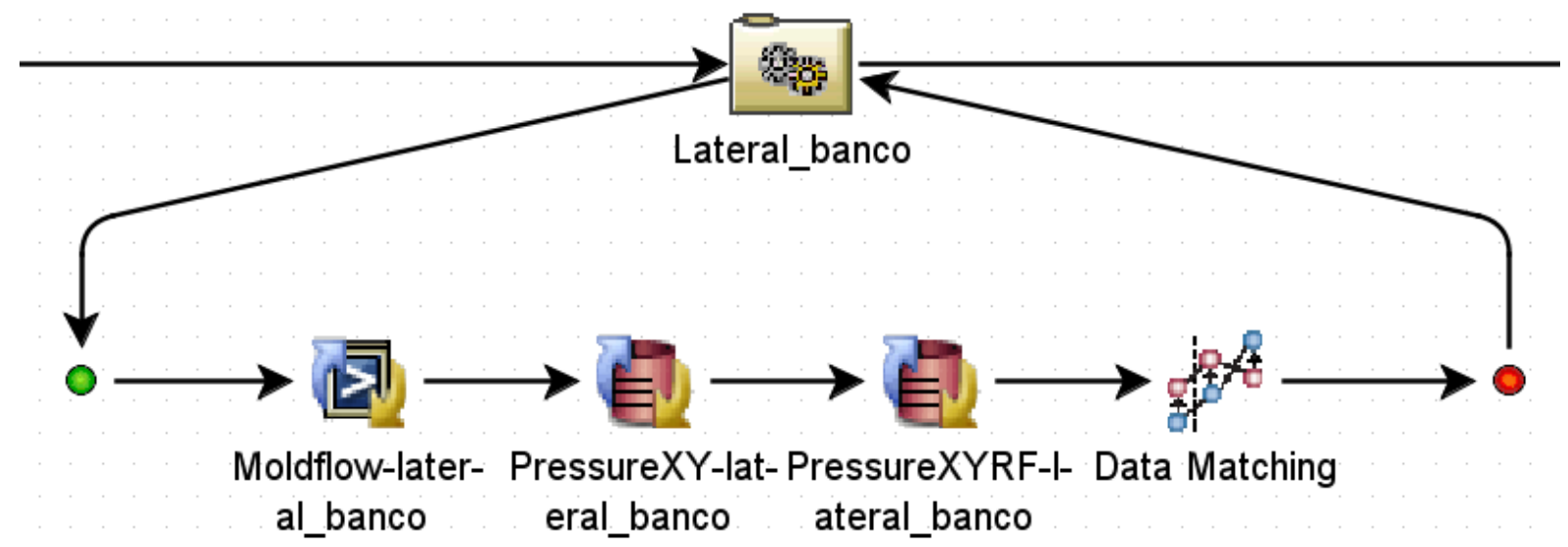

Figure 8. Tela do programa Isight - workflow do processo de simulação de injeção.

Após a obtenção dos critérios para ambas as análises tem um componente "calculator" que calcula a média aritmética dos critérios. 


\subsection{DOE}

Para definir os experimentos a serem realizados e estudar os dados obtidos foi utilizado o método de delineamento de experimentos "Optimal Latin Hypercube". O objetivo desse método é distribuir de maneira aleatória os pontos de experimentos garantindo uma distância uniforme entre os pontos estudados.



Figure 9. Exemplo da distribuição de experimentos no método "Optimal Latin Hypercube".

Foram utilizados 100 experimentos sendo que os coeficientes alterados e os níveis utilizados foram realizados de acordo com a figura 10:

\begin{tabular}{|c|c|c|c|c|c|c|}
\hline 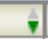 & Parameter & Lower & Upper & Relation & Baseline & Values \\
\hline$\checkmark$ & $\bullet$ D1 & $1.0 \mathrm{E} 10$ & $1.0 \mathrm{E} 20$ & values & $1.0 \mathrm{E} 15$ & 1.0E10 1010101019999... \\
\hline$\checkmark$ & $\bullet$ D2 & 113.2 & 263.15 & values & 260.97 & $263.15260 .97113 .226 \ldots$ \\
\hline$\checkmark$ & $\bullet$ Tau & 0.0 & 15000.0 & values & 7500.0 & $0.0151 .5152303 .0303 \ldots$ \\
\hline$\checkmark$ & $\bullet \mathrm{n}$ & 0.1 & 0.6 & values & 0.38 & $0.10 .1050 .110 .1150 .1 \ldots$ \\
\hline$\checkmark$ & $\bullet A 2$ & 51.6 & 211.55 & values & 51.6 & $51.6211 .5551 .6211 .55 \ldots$ \\
\hline$\checkmark$ & $\bullet A 1$ & 10.0 & 85.0 & values & 15.0 & $10.010 .511 .011 .512 .0 \ldots$ \\
\hline
\end{tabular}

Figure 10. Tabela de parâmetros utilizados.

O coeficiente "D3" não foi alterado porque para todos os poliproprilenos encontrados no banco de dados do programa ASMI esse coeficiente é igual à 0.

\section{RESULTADOS E DISCUSSÃO}

Foi possível identificar que os fatores de maior influência em relação aos critérios de comparação foram os parâmetros n e D2, sendo que a influência do parâmetro D2 não é linear, conforme se observa no gráfico de Pareto e nos gráficos de efeito principal.

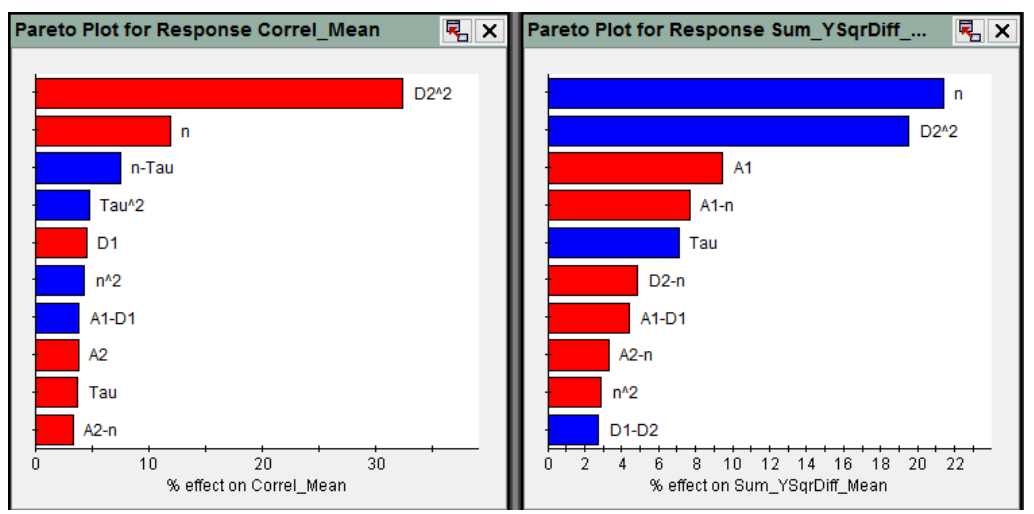

Figure 11. Gráfico de Pareto a partir dos resultados obtidos no DOE. 


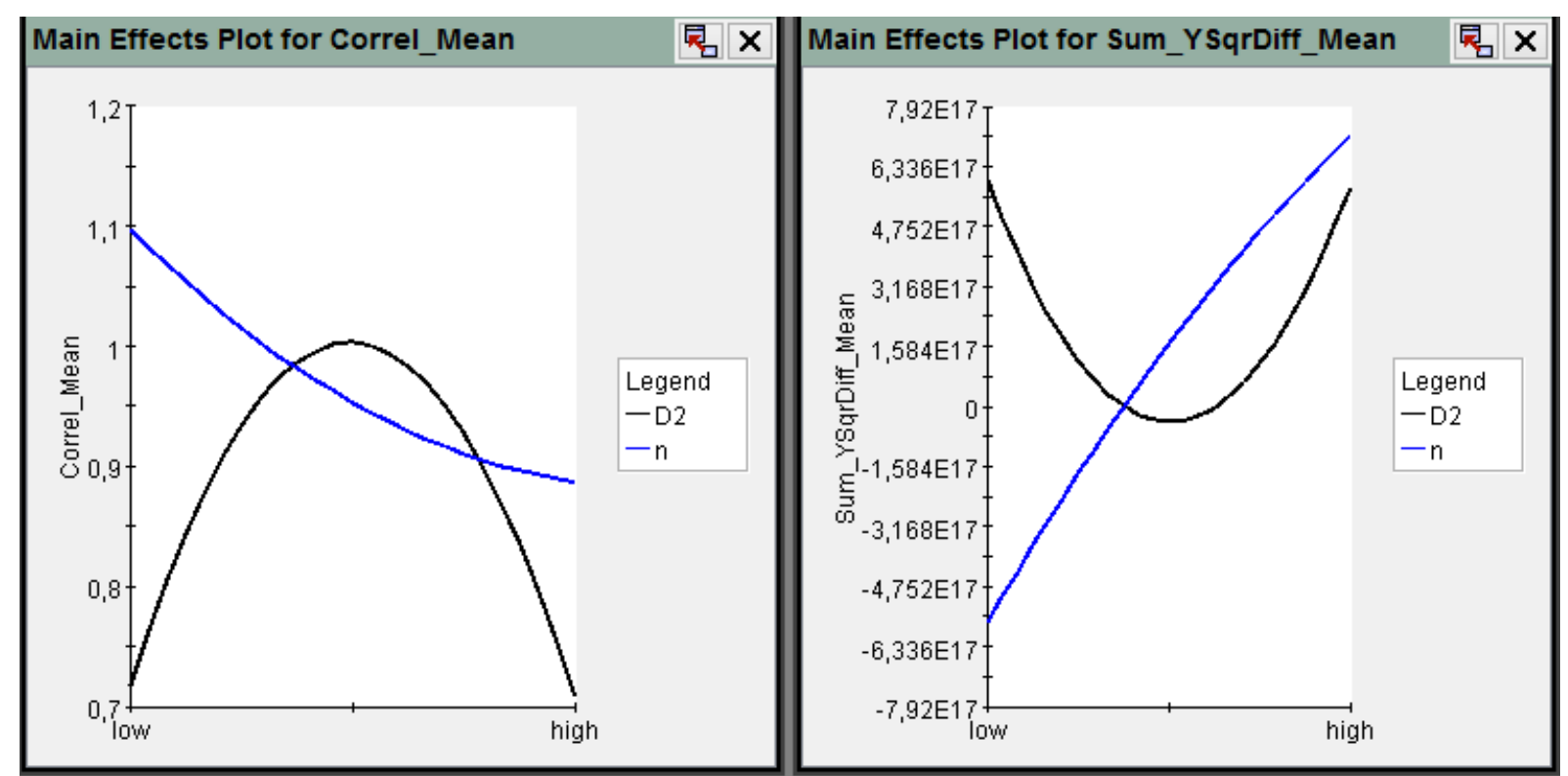

Figure 12. Gráfico de principal interação dos parâmetros n e D2.

Outro ponto é que a influência dos parametros varia conforme os critérios utilizados. Desse modo, quando avaliado o critério de correlação não há grande interação entre os parametros n e D2. Por outro lado, quando se avalia a soma do quadrado da diferença, $n$ e D2 apresentam uma grande interação de efeitos, como pode ser visto no gráfico da figura 13.

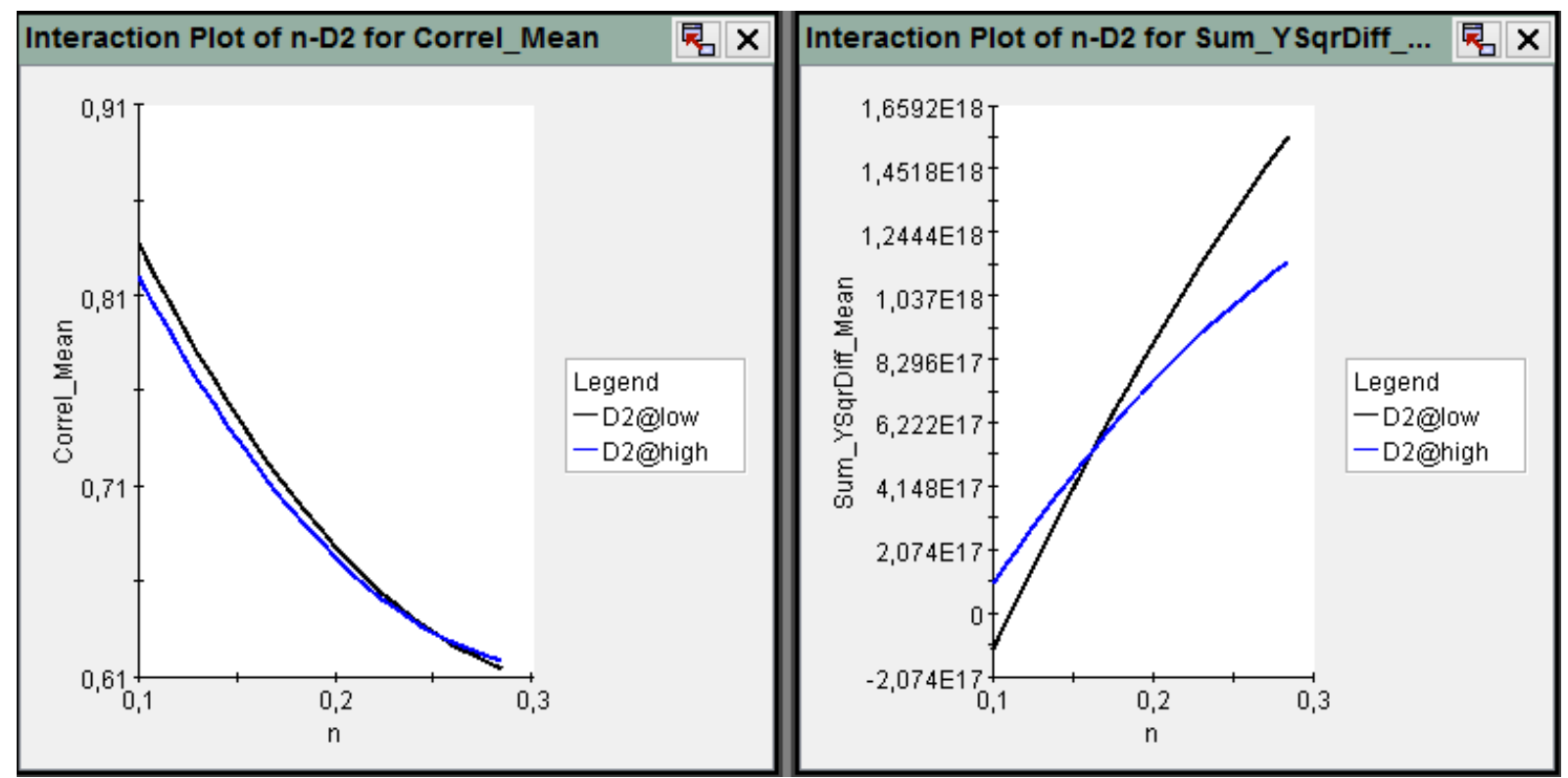

Figure 13. Gráfico de interação entre os parâmetros n e D2.

O programa Isight gera uma tabela com os resultados de todos os experimentos e destaca aquele que apresenta melhores resultados em função dos critérios definidos. Desse modo, obtemos o material a ser utilizado no programa ASMI. 


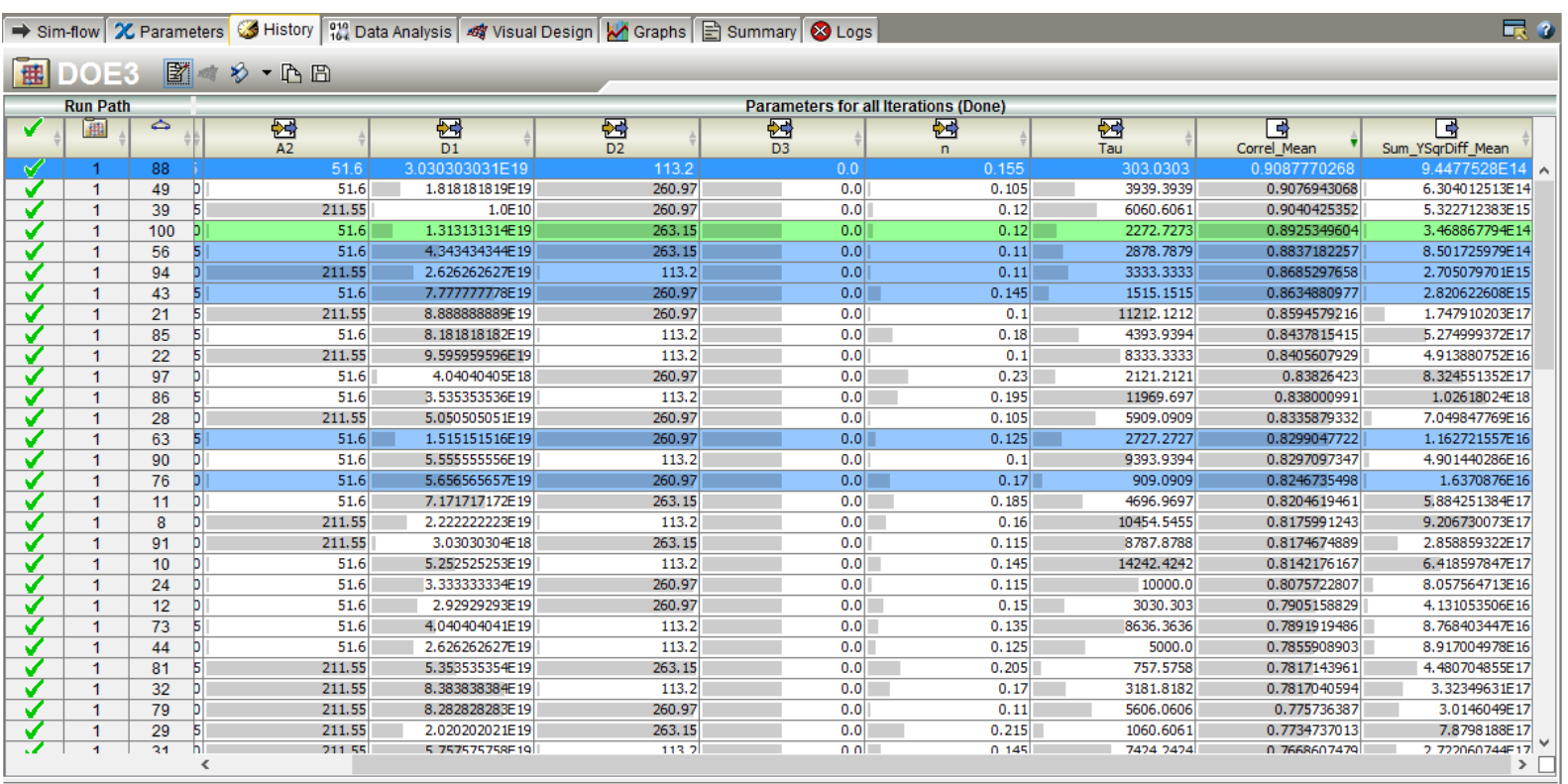

Figure 14. Tabela de resultados dos experimentos

Um arquivo *.UDB foi gerado contendo as propriedades materiais obtidas neste processo de calibração e os resultados da simulação foram comparados com os resultados obtidos de simulação com propriedades obtidas em laboratório pelas técnicas convencionais.

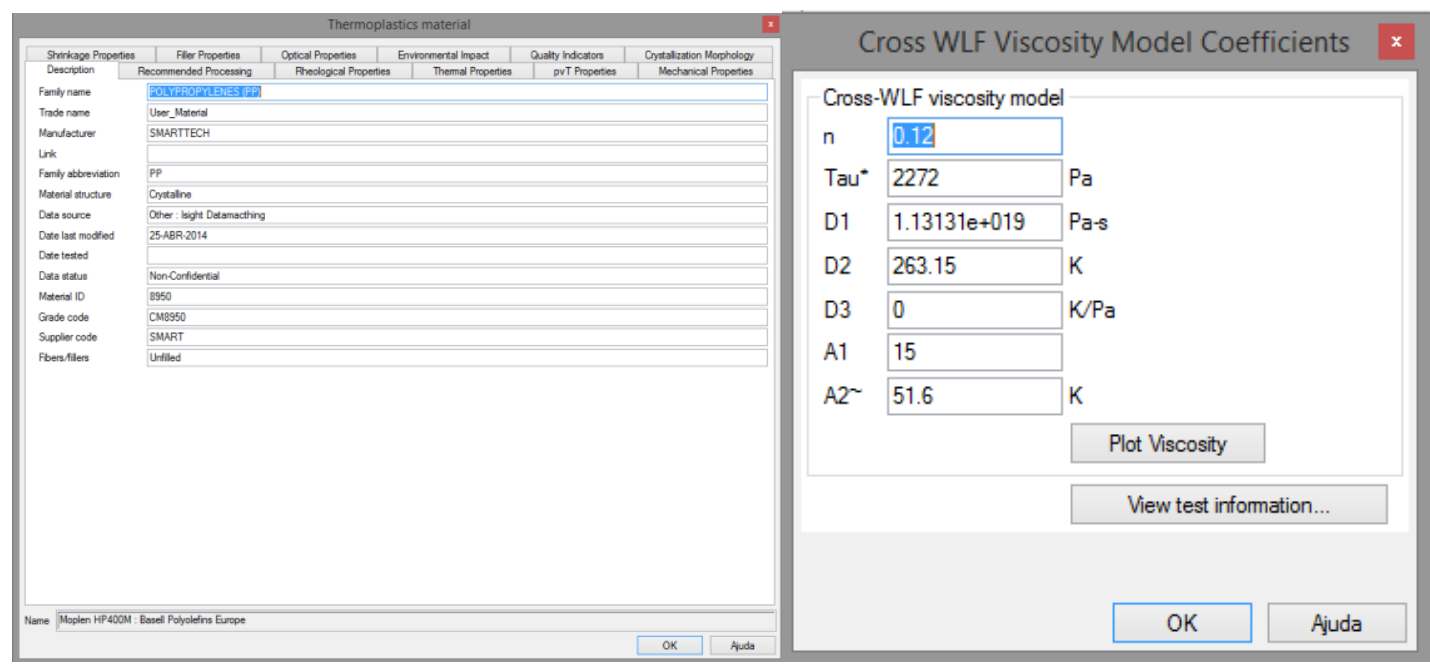

Figure 15. Resumo das informações e coeficientes do material obtido

Comparando os resultados de preenchimento, o material gerado a partir dos dados obtidos no processo de calibração forneceu resultados bastante próximos para os dois estudos, sendo que o material proposto apresenta uma fluidez um pouco maior que o material obtido via laboratório. 


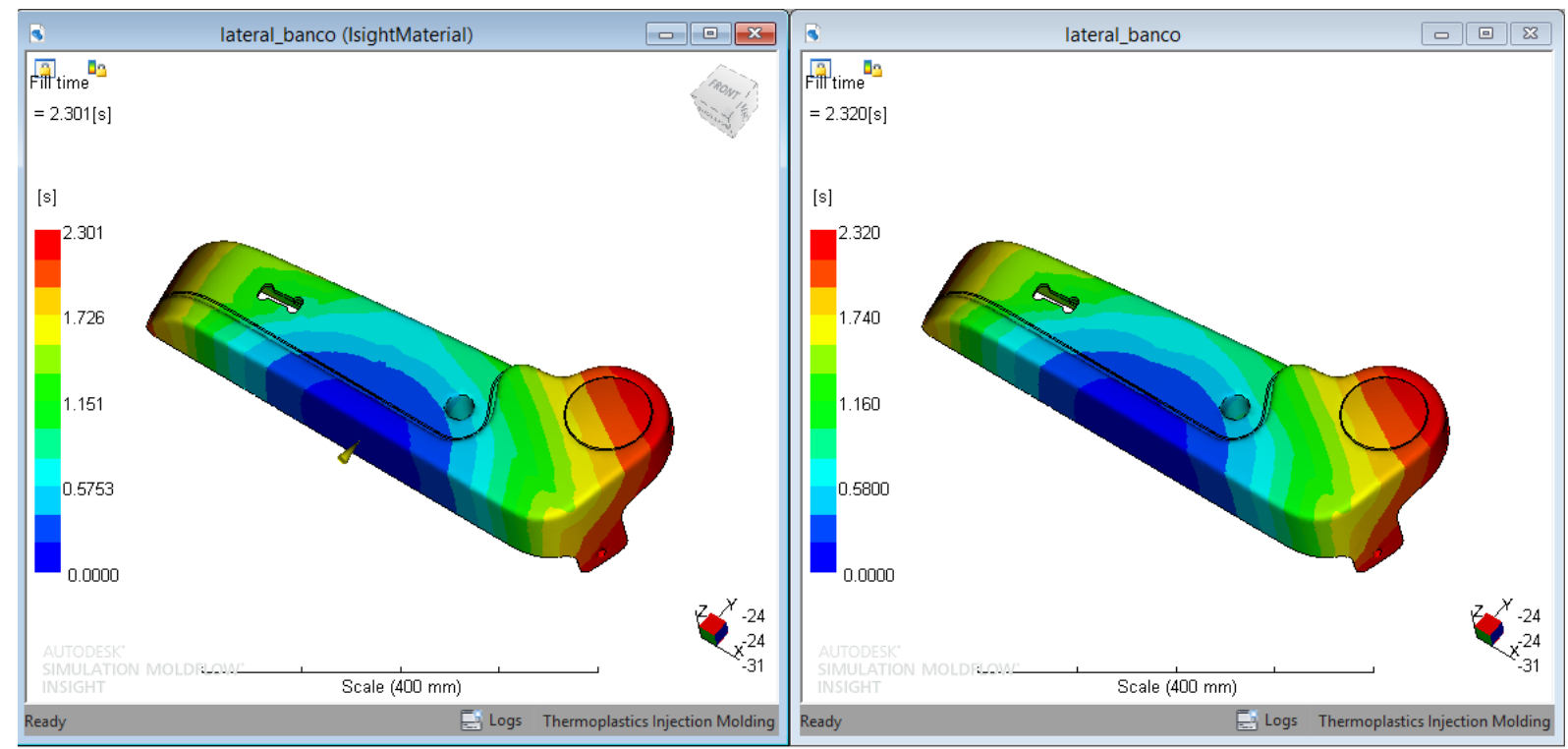

Figure 16. Gráfico de preenchimento da peça Lateral de Banco do programa ASMI

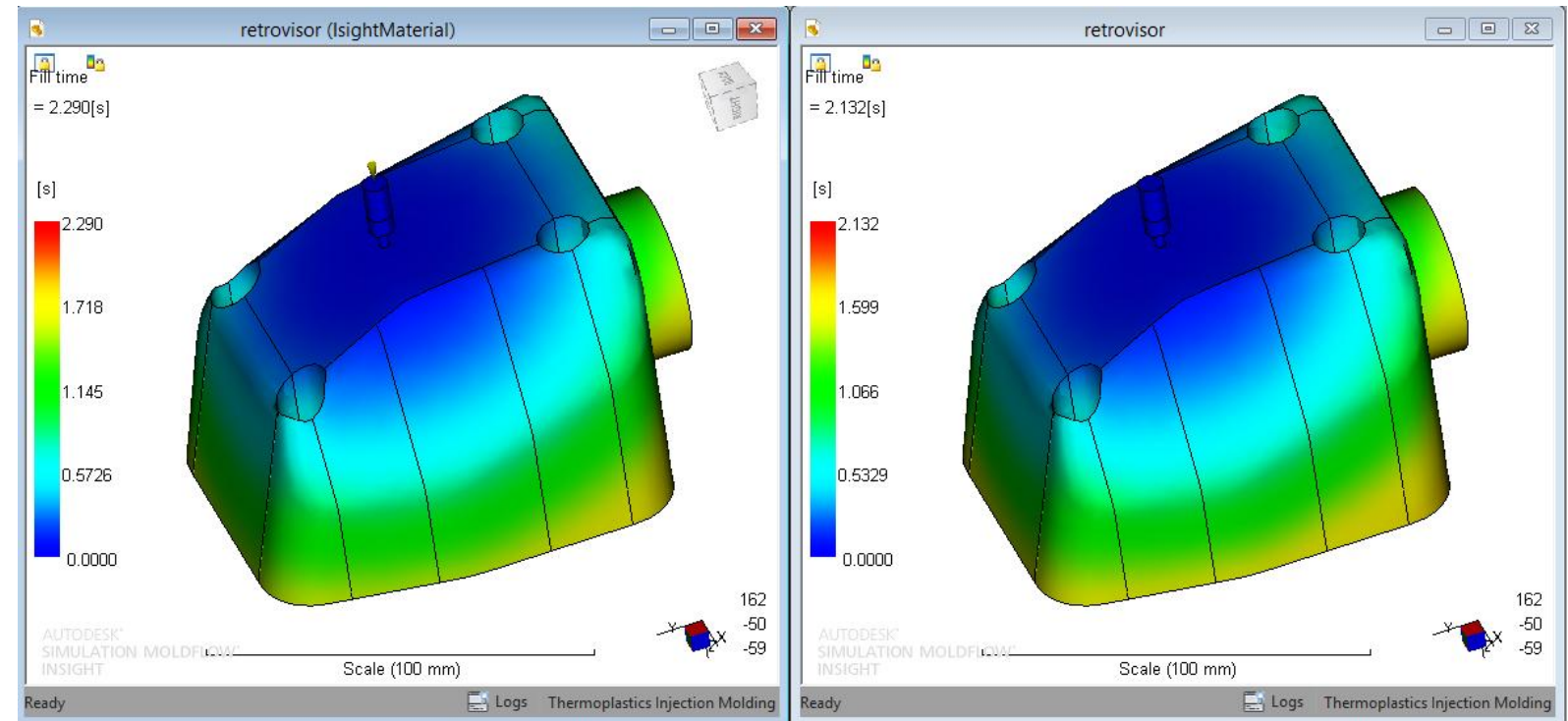

Figure 17. Gráfico de preenchimento da peça Retrovisor do programa ASMI

A distribuição de pressão também se mostrou bastante próxima, sendo em torno de $10 \%$ de diferença e distribuição similar ao longo do preenchimento [6]. 

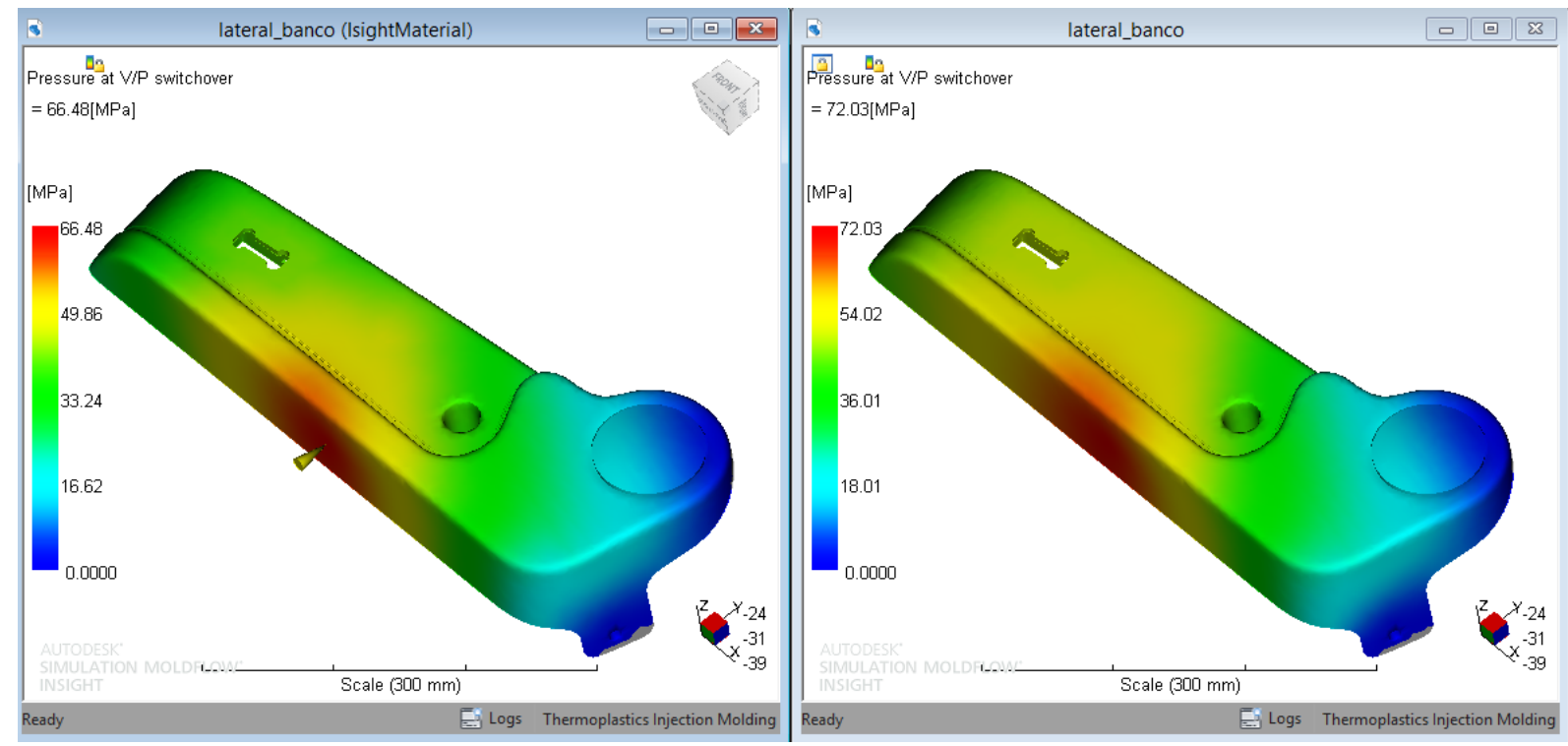

Figure 18. Gráfico de pressão no momento da comutação da peça Lateral de Banco do programa ASMI

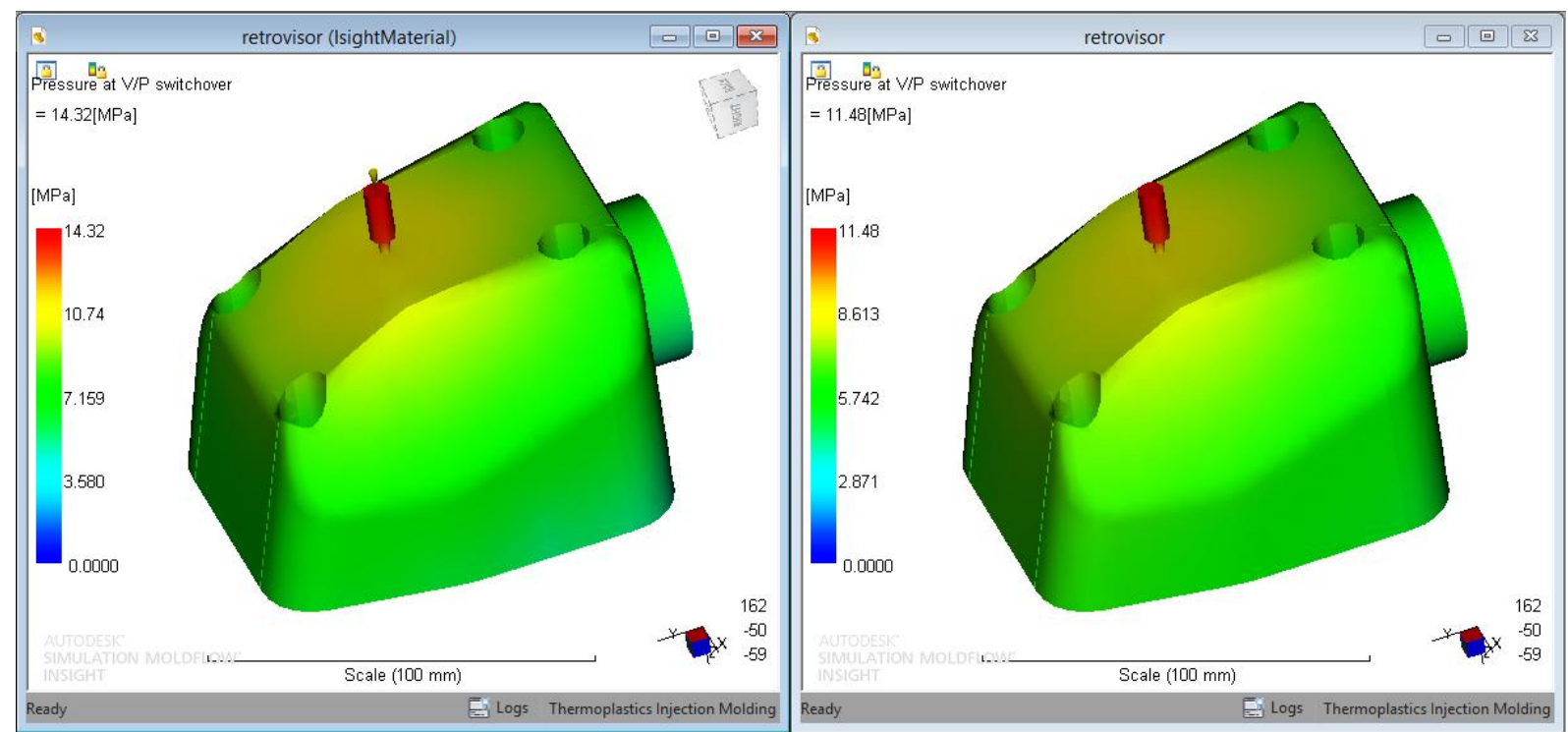

Figure 19. Gráfico de pressão no momento da comutação da peça Retrovisor do programa ASMI

Fazendo a comparação na curva de pressão pode-se avaliar que as curvas têm a mesma tendência. Para a peça lateral de banco, apenas o valor máximo de pressão divergiu em $8 \%$, ao passo que, para o caso da peça retrovisor a curva apresenta a mesma tendência, porém com valores menores. 


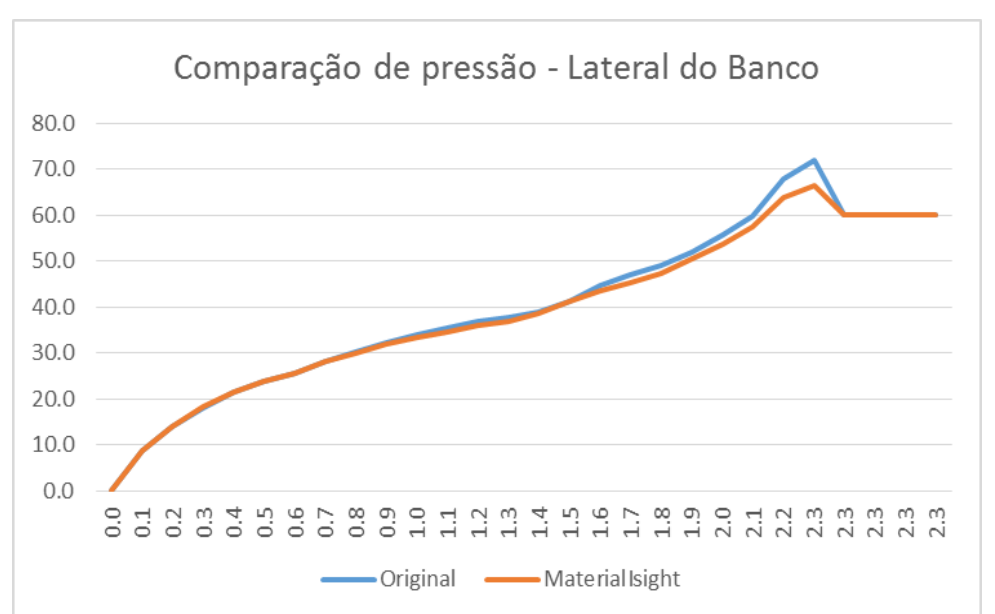

Figure 20. Gráfico de pressão durante o preenchimento do material original (Moplen HP400M) e do material obtido via calibração do modelo da peça Retrovisor



Figure 21. Gráfico de pressão durante o preenchimento do original (Moplen HP400M) e do material obtido via calibração do modelo da peça Lateral do Banco

Comparando as curvas de viscosidade encontradas, pode-se notar que os valores encontrados não foram suficientes para descrever o comportamento da viscosidade para diferentes temperaturas. Isto pode ter ocorrido em virtude dos experimentos avaliados não possibilitarem uma calibração do modelo para diferentes temperaturas e sim um único processo para cada geometria.


Figure 21. Gráfico de viscosidade do material original (Moplen HP400M) com o Material obtido na calibração do modelo. 


\section{CONCLUSÃO}

A metodologia estudada nesse trabalho mostrou-se válida para obter os coeficientes de viscosidade a partir da curva de pressão obtidos para duas geometrias distintas. Desde modo, apresenta-se como uma alternativa de menor custo e mais acessível para caracterização de material para simulação do processo de injeção. Outro ponto positivo da metodologia é a possibilidade de estudar os impactos das características do material no processo de injeção.

Como próximo passo, deverá ser avaliada a possibilidade de realizar estudos com diferentes processos para a mesma geometria, a fim de representar os impactos da variação de temperatura nas curvas de viscosidade, aproximando o resultado obtido do ideal além de estender a pesquisa à calibração de outros coeficientes, como à curva de PVT e aos dados de contração.

\section{REFERÊNCIAS}

[1] OLIVON, Beatriz; O que o Inova-Auto quer das empresas. Disponível em http://exame.abril.com.br/economia/noticias/o-que-o-inovar-auto-quer-das-empresas?page=2 Acesso em: 21/03/2014.

[2] FRANCISCO, Anderson da Silva. Otimização do ciclo pelo processo de moldagem por injeção convencional. 74 páginas, Monografia (Graduação). Faculdade de Tecnologia da Zona Leste.

[3] SHOEMAKER, Jay; Moldflow Design Guide: a Resource for Plastic Engineers. Framingham, MA: Hanser Publications, 2006.

[4] KAMAL, Musa R.; ISAYEV, Avraam; LIU, Shih-Jung; Injection Molding:Technology and Fundamentals. Munich: Hanser Publishers, 2009

[5] KENNEDY, Peter Kenneth; Practical and Scientific Aspects of Injection Molding Simulation. 143 páginas, Dissertação (Doutorado). Technische Universiteit Eindhoven

[6] Autodesk Knowledge Network; Autodesk Simulation Moldflow Insight 2015 - Help, Disponível em http://help.autodesk.com/view/MFIA/2014/ENU/?guid=GUID-5F3A6D607140-4389-B35C-C03DE61B2041 Acesso em: 08/05/2014. 